\title{
Chronic NMDA Receptor Blockade from Birth Increases the Sprouting Capacity of Ipsilateral Retinocollicular Axons without Disrupting Their Early Segregation
}

\author{
Matthew T. Colonnese ${ }^{1}$ and Martha Constantine-Paton ${ }^{2}$ \\ 1/nterdepartmental Neuroscience Program, Yale University, New Haven, Connecticut 06520, and 2Department of Biology, \\ Massachusetts Institute of Technology, Cambridge, Massachusetts 02139-4307
}

\begin{abstract}
We have investigated the role of the NMDA glutamate receptor (NMDAR) in the genesis and regulation of structural plasticity during synaptogenesis in the visual layers of the rat superior colliculus (sSC). In this neuropil, three projections compete for synaptic space during development. By fluorescently labeling the projections of both eyes and imaging them with confocal microscopy, we can quantify the sprouting of the ipsilateral retinal projection that follows removal of a portion of the contralateral retinal and/or corticocollicular projection. Using these techniques we have studied the effects of NMDAR blockade under different levels of competition. NMDARs were chronically blocked from birth [postnatal day 0 (P0)] by suspending the competitive antagonist 2-amino-5-phosphonopentanoic acid in the slow release plastic Elvax, a slab of which was implanted over the sSC. Such treatment alone does not impair the normal
\end{abstract}

segregation of the retinal projections. However, if sprouting of the ipsilateral projection is initiated with a small contralateral retinal lesion at P6, this sprouting can be further increased by blocking NMDARs from birth. Sprouting of the ipsilateral retinal projection is also induced by retinal lesions made at P10/P11, but NMDAR blockade does not augment the sprouting induced by this later lesion. However, when combined with simultaneous ablation of the ipsilateral visual cortex, P10/P11 lesions show increased sprouting after NMDAR blockade. These data indicate that PO NMDAR blockade does not eliminate synaptic competition in the sSC. Instead, early elimination of NMDAR function appears to facilitate sprouting that is gated in a stepwise manner by the other visual afferents.

Key words: development; glutamate receptors; sprouting; superior colliculus; retina; rat
Neuronal development is marked by the passing of a number of critical periods for plasticity; one of the most dramatic is the ability of afferents to sprout outside their normal terminal zones to compensate for a lesion (Lund, 1978). Recent work has shown that factors within the neuropil act to prevent sprouting and when these factors are removed axons retain substantial sprouting ability (Bandtlow and Schwab, 2000). Synaptic factors may also affect the sprouting response. Defining which receptor systems are involved will not only help facilitate the return of function after trauma but also begin to provide the link between physiologically defined plasticity and structural change.

The NMDA glutamate receptor (NMDAR) is a critical component of numerous forms of developmental (Cline et al., 1987; Kleinschmidt et al., 1987; Udin and Scherer, 1990; Schlaggar et al., 1993) and adult (Garraghty and Muja, 1996) plasticity. Downregulation of the NMDAR has been implicated in the end of ocular dominance plasticity (Daw, 1995), as well as the loss of long-term potentiation (Kirkwood et al., 1995). However, although important for physiological plasticity and a necessary component of some structural refinements that occur in the developing retinof ugal pathways (Hahm et al., 1991; Simon et al., 1992; Ernst et al., 1999), it is still not clear whether NMDARs are

Received July 26, 2000; revised Nov. 20, 2000; accepted Nov. 28, 2000.

This work was supported by National Institutes of Health Grant EY06039 to M.C.-P. and National Eye Institute Grant EY07115 to M.T.C.

Correspondence should be addressed to Dr. Martha Constantine-Paton, Building 68-380, Massachusetts Institute of Technology, 77 Massachusetts Avenue, Cambridge, MA 02139-4307. E-mail: mcpaton@mit.edu.

Copyright (C) 2001 Society for Neuroscience 0270-6474/01/211557-12\$15.00/0 critical for interpathway competition, the removal of which results in pronounced structural rearrangements.

We are investigating this question using the superficial visual layers of the developing rat superficial superior colliculus (sSC), where it is possible to study developmental changes in the ability of axons to sprout after the physical removal of competition. The rodent $\mathrm{SSC}$ is an attractive system because functional changes in synaptic currents occur quickly and are synchronized across most cell types (Shi et al., 1997). Previous work by Lund et al. (1973) on the retinocollicular system showed that neonatal eye removal causes substantial elaboration of the axons of the remaining eye into the deafferented region, but only when the ablation occurred before postnatal day 10 (P10). We were intrigued by a potential link between this competitive interaction and NMDAR function because our work has demonstrated a pronounced drop in NMDAR current decay time between P10 and P11 (Shi et al., 1997).

We have used chronic blockade of NMDARs from birth to explore their role in the sprouting of the ipsilateral retinal projection. One of the technical issues in working with this projection is the variability of its sSC innervation even in normal animals. Consequently we have developed a quantitative method of assaying the density of the ipsilateral projection after lesion of the competing retinal and/or cortical projections. Intraocular injection of fluorescently tagged cholera toxin B-subunit tracers (CTB) allowed visualization of both retinal projections, whereas confocal microscopy enabled objective quantification of the density of these projections in a thin tissue section.

Our results indicate that NMDARs are not necessary for competition among the glutamatergic projections to the sSC. 
However, after lesion of competing projections, NMDAR blockade significantly increased the sprouting of the ipsilateral retinal projection in a manner proportional to the level of competition removed.

\section{MATERIALS AND METHODS}

Animals and materials. Timed pregnant Sprague Dawley rats were acquired from CAMM (Wayne, NJ) or Charles River Laboratories (Southbridge, MA) and housed on a 12/12 hr light/dark cycle. To block NMDARs chronically, the inert ethylene-vinyl acetate copolymer Elvax40W (DuPont, Billerica, MA) was prepared as reported previously (Simon et al., 1992; Prusky and Ramoa, 1999). A $1 \mathrm{~mm}$ solution of the racemic mixture (+/-)-2-amino-5-phosphonopentanoic acid (AP5; Sigma, St. Louis, MO) or, as a control, a $500 \mu \mathrm{M}$ solution of the inactive isomer L-2-amino-5-phosphonopentanoic acid (L-AP5) was prepared in a $10 \%$ Elvax solution in methylene chloride (AP5-Elvax and L-AP5-Elvax, respectively). The solvent was evaporated at $-20^{\circ} \mathrm{C}$, and the resulting plugs were lyophilized overnight before being cut $180 \mu \mathrm{m}$ on a cryostat. For anterograde tracing of the retina, $\mathrm{CTB}$ conjugated to a tetramethyl rhodamine B (tRITC) or fluorescein (FITC) fluorophore (List Biological Laboratories, Campbell, CA) was made in a $0.2 \%$ solution in $1 \%$ DMSO.

Surgery. Elvax sections were implanted at P0 using the method of Simon et al. (1992); under hypothermic anesthesia, the scalp and skull were retracted, and the Elvax slab was placed over the sSC, the front edge tucked under the occipital cortex. Sutures and tissue adhesive (Vetbond; 3 M Animal Care Products, St. Paul, MN) were used to close the incision, and the pups were warmed under a lamp until ready to be returned to the mother. This method has been estimated to release water-soluble drug in the range of picomoles per day (Cline and Constantine-Paton, 1989; Smith et al., 1995). Because the rate of clearance is unknown, the actual concentration in tissue is difficult to estimate. Recordings under the Elvax in vivo have demonstrated substantial blockade of all glutamatergic activity when the concentration of AP5 in Elvax is 20 times that in our experiments (Schlaggar et al., 1993). On the basis of all these data, we estimate our concentration of D-AP5 in the sSC to be $>20 \mu \mathrm{M}$ at all times and in the $100 \mu \mathrm{M}$ range during the first few days after implantation.

For eye lesions, pups were anesthetized with a 2-bromo-2-chloro-1,1,1triflurooethane (halothane) vaporizer (Cyprane "Fluotec"), and the left eye was opened with blunted iris scissors. The eyelids were retracted manually, and the lesion was made by lightly touching a heat microcauterizer to the sclera adjacent to the ciliary margin. Under the same anesthesia, the animals that were to receive a cortical lesion in addition to the eye lesion had the scalp retracted on the right side. A small rectangular section $(1 \times 4 \mathrm{~mm})$ of skull was removed over the occipital lobe, and the underlying dura was cut. The occipital cortex was aspirated with a glass pipette, the evacuated region was filled with sterile Gelfoam (Upjohn, Kalamazoo, MI), and the incision was closed as described above. For anterograde labeling, 1 or $2 \mathrm{~d}$ before death, pups were anesthetized with inhaled 1-chloro-2,2,2-trifluoroethyl difluoromethyl ether (isoflurane). With a 28 gauge needle, a small hole was made in the cornea through which a Hamilton syringe was used to inject $5 \mu \mathrm{l}$ of the CTB solution into the vitreous.

Under isoflurane anesthesia, pups were killed by transcardial perfusion of PBS followed by $4 \%$ paraformaldehyde in $0.1 \mathrm{M}$ phosphate buffer, $\mathrm{pH}$ 7.2. The midbrain and eyes were removed and post-fixed overnight at $4^{\circ} \mathrm{C}$. Pups with misplaced Elvax or damage to the sSC were excluded from further analysis. The midbrain was blocked and photographed in wholemount epifluorescence at $1 \times[0.04$ numerical aperture (NA) Planar lens on a Nikon E600 equipped with a CCD camera (Diagnostic Instruments, Inc., Sterling Heights, MI)] to visualize the dorsal midbrain optic targets. Pups with a lightly or incompletely labeled projection from the unlesioned eye or with a scotoma (deafferented region) that occupied $>35 \%$ or $<7 \%$ of the sSC were excluded from further analysis. The midbrain blocks were embedded in $3.5 \%$ low-melting point agarose (Sigma) and $8 \%$ sucrose in PBS and cut in coronal sections $100 \mu \mathrm{m}$ thick on a vibratome. Sections were mounted on glass slides and coverslipped in a hardening aqueous medium (EM Sciences, Fort Washington, PA).

Quantification of sprouting. All confocal microscopy was performed on a Bio-Rad (Hercules, CA) 1024 MRC through a $25 \times, 1.20$ NA plan-apo objective with simultaneous two-color fluorescence microscopy. Gain, black level, and pinhole size were kept constant for each litter, and the examination of pups from each treatment group was interdigitated to avoid problems caused by slow shifts in laser power or alignment. Frames were selected for analysis by a regular sampling scheme that was similar for lesioned and unlesioned pups, although the area sampled was more expansive for unlesioned pups. For them, alternate sections were selected across the entire rostral-caudal axis of the sSC, and four evenly spaced micrographs were taken from each section. Of these four frames, two were from the medial and lateral edges of the sSC, and the remaining two were evenly spaced within the crown, at least $500 \mu \mathrm{m}$ from the edges. For the rostral pole analysis of normal animals, only the two sections from the crown were used. These were taken from three alternate sections beginning $400 \mu \mathrm{m}$ from the rostral edge. For the quantitative analysis of retinal ganglion cell axon density in lesioned animals, three alternate sections were chosen from each animal: the first, $\sim 400 \mu \mathrm{m}$ from the rostral edge, and two alternate sections thereafter. Two micrographs were taken from each section: the first outside the scotoma and at least $500 \mu \mathrm{m}$ from the medial edge (see Fig. $4 D, E$ ) and the second within the scotoma and at least $200 \mu \mathrm{m}$ from the lateral edge (see Fig. $4 D, F$ ).

Regardless of sampling scheme, each confocal micrograph consisted of a $z$ series of three to five confocal images taken at $5 \mu \mathrm{m}$ intervals. Image analysis was performed off-line on a Macintosh G3 computer using the public domain NIH Image program (developed at the National Institutes of Health and available on the internet at http://rsb.info.nih.gov/nihimage/). The density of the ipsilateral retinal projection was calculated for each confocal $z$ section by creating a binary image with a threshold value chosen so that only pixels from in-focus fluorescence were retained. This threshold was set after examination of multiple pups in a litter and was maintained for all animals in that litter. The threshold was approximately sixfold greater than the level of tissue autofluorescence, the exact number varying between animals and litters because the baseline autofluorescence varies slightly. The thickness of each optical section was $\sim 2-3 \mu \mathrm{m}$.

By use of the contralateral projection as a guide, the area of the middle and upper stratum griseum superficiale (SGS) and stratum zonale (SZ), together designated SGS/SZ, or the stratum opticum (SO) was defined for each $z$ section. For each frame the density of labeled pixels was measured in the defined layers. These densities were separately obtained for both the SGS/SZ and SO for each $z$ section in a frame, and the average intensity from all of the $z$ sections in the frame was calculated for the SGS/SZ and SO. This number was designated the "labeled pixel density," and it is an objective estimate of the innervation density of the ipsilateral retinal ganglion cell axons in the region of interest.

To quantify sprouting of the ipsilateral projection into a contralateral retinal scotoma, labeled pixel densities were generated from the SGS/SZ from frames selected by use of the sampling regimen described above. Thus, for each animal, we generated three pairs of labeled pixel density measurements. Because each pair was from the same section, there was an internal control for each position along the rostral-caudal axis. To estimate the additional ipsilateral axon density that was caused by the lesion, for each section we calculated an "increased labeled pixel density" by subtracting the labeled pixel density of the frame outside the scotoma ([Out]) from the labeled pixel density of the frame inside the scotoma ([In]). To normalize for comparison between litters in which a similar experimental procedure was performed, each treatment was applied to half the pups in a single litter. After data collection, the increased labeled pixel density of each section was divided by the average value of the increased labeled pixel densities in the sections taken from the control pups in the same litter. Therefore, the equation for the normalized increased labeled pixel density for each section looks like this:

$$
[\mathrm{In}]_{x}-[\mathrm{Out}]_{x} /\left(\sum\left([\mathrm{In}]_{\text {control }}-[\text { Out }]_{\text {control }}\right) / N_{\text {control }}\right)
$$

where $[\mathrm{In}]_{X}$ and [Out $]_{X}$ are the labeled pixel densities for a given section $X$ and $N_{\text {control }}$ is the number of control sections; the subset of labeled pixel densities from control animals are denoted $[\mathrm{In}]_{X \text { control }}$ and $[\mathrm{Out}]_{X^{-}}$ control. For statistical analysis, all normalized increased labeled pixel densities were pooled by treatment type for each experiment. All statistics are from a two-tailed $t$ test unless otherwise noted, and the error bars represent the SEM.

For immunohistochemistry, rats were lesioned, labeled, and killed as described above. After fixation, the blocked midbrains were cryoprotected in $30 \%$ sucrose in PBS, frozen on dry ice, and cut $30 \mu \mathrm{m}$ on a cryostat. The floating sections were exposed to $5 \%$ donkey serum and $0.5 \%$ Triton X-100 in PBS for $1 \mathrm{hr}$, followed by monoclonal synaptophysin primary antibody (Sigma; $1: 500$ ) in the same solution overnight at $4^{\circ} \mathrm{C}$. Secondary antibody conjugated to the Cy5 fluorophore (Jackson ImmunoResearch, West Grove, PA) was used for visualization. 
Figure 1. Simultaneous labeling of eyes with fluorophore-conjugated CTB allows detailed imaging of both retinocollicular projections. A, Flat mount of a P14 retina that has been injected with FITC CTB. Retinal ganglion cell axons stream to the optic disk evenly from all quadrants. $B, C$, Montages of confocal micrographs from a single hemisphere from the rostral region of a P14 rat sSC cut in coronal section. Each section is a brightest pixel projection of a $20-\mu \mathrm{m}$ deep $z$ series. The contralateral $(B)$ and ipsilateral $(C)$ retinal afferents have been labeled by intraocular injection of CTB conjugated to tRITC or FITC, respectively, and were imaged simultaneously through two channels. The contralateral projection forms a dense sheet in the SGS and SZ $(B)$, whereas the ipsilateral projection is mostly confined to the SO, with denser SGS projections $\mathrm{n}$ the medial and lateral edges $(C)$. Refined arbor patches can be seen (arrows)
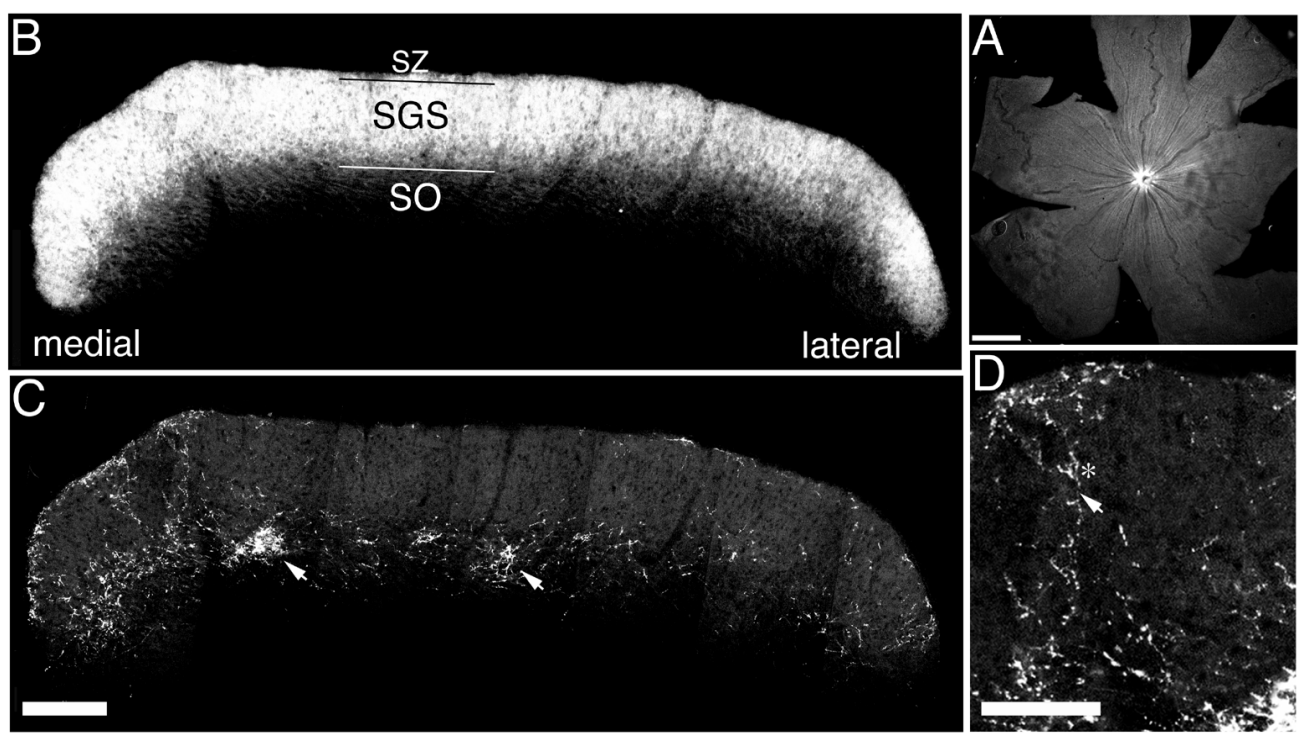

at this age. SZ, SGS, and SO refer to the layers of the visual sSC. $D$, Detail of $C$ showing the structure of an ipsilateral arbor. Label is dominant in the arbors (asterisk) although the axons are still visible (arrow). Scale bars: $A, 1 \mathrm{~mm} ; B, C, 200 \mu \mathrm{m} ; D, 100 \mu \mathrm{m}$.

\section{RESULTS}

Retinal ganglion cell axons in the midbrain were visualized with anterograde transport of fluorophore-conjugated CTB (Fig. 1). When injected into the vitreous of the eye, ganglion cell axons were labeled evenly throughout the retina, and their axons formed a dense layer of arborization in the contralateral sSC, particularly the SZ and the upper and middle SGS (Fig. 1B). In the sparser ipsilateral projection, the structure of the axon arbors could be observed (Fig. 1C). The majority of tracer fluorescence is from arbors and axon swellings, with lighter label in the axon proper (Fig. 1D, asterisk, arrow, respectively). By simultaneously labeling both eyes with different fluorophore-conjugated tracers, the distribution of the ipsilateral projection could be examined in relation to the gross distribution of the contralateral projection. We observed an adult-like pattern of arborization by the time of eye opening at P14 (Land and Lund, 1979). In the rostral pole of the sSC, the ipsilateral retinal projection was mostly restricted to the upper SO, with sparse but substantial arborizations in the medial and lateral upper SGS (Fig. 1C). Within the SO the ipsilateral projection had, by $\mathrm{P} 14$, formed the scattered dense patches of arborization characteristic of its refined state (Fig. 1C, arrows).

\section{PO NMDAR blockade does not alter the normal refinement of the ipsilateral retinocollicular projection}

We first determined whether the normal projection pattern of the ipsilateral retina was disrupted by chronic NMDAR blockade. To apply the competitive antagonist AP5, we used thin slabs of the slow-release plastic Elvax implanted above the sSC at birth. As a control, other pups received Elvax in which only the inactive stereoisomer L-AP5 was suspended. We detected no qualitative differences in ipsilateral retinal arborization between AP5-Elvaxtreated, L-AP5-Elvax-treated, or untreated pups at P14 (Fig. 2). The projection had normally withdrawn from the caudal region of the sSC (data not shown) as well as from the rostral SZ and SGS (Fig. 2B). Of particular note is the persistence of arbor patches in the SO, the formation of which has been shown to be dependent on the activity of retinal ganglion cells (Thompson and Holt, 1989) as well as on normal function of their terminals (Mooney et al., 1998). To quantify the refinement of the ipsilateral projection we measured the labeled pixel density of ipsilateral axons and arbors in confocal $z$-section micrographs from the SZ/SGS or SO (see Materials and methods). This statistic reflects the twodimensional density of the ipsilateral retinal projection from a depth of 2-3 $\mu \mathrm{m}$. To minimize variability, a single litter was divided into three groups (three pups each): normal pups that received no surgery and one group each with either AP5-Elvax or L-AP5-Elvax implanted on the day of birth. No differences between the AP5-treated group and either of the control groups were found at any position on rostral — caudal and/or mediallateral axes (data not shown; comparison by ANOVA, all $p$ values $>0.05$ ). For comparison with the results in the remainder of this study, we were particularly interested in the rostral regions of the sSC, where temporal retinal lesions will form a scotoma. In Figure $2 C$ we present the average labeled pixel density measured from two micrographs from each of three sections per pup. These regions were selected in a manner identical to that of subsequent groups with a contralateral temporal retina lesion (see Materials and Methods). One micrograph is from the region that would be outside the scotoma, and the other is from the region that would be inside it. ANOVA showed no difference among any treatment groups (SZ/SGS, $F=0.27 ; p=0.76$; $\mathrm{SO}, F=1.03 ; p=0.36$ ). The two micrographs from each section were approximately from regions that would be inside or outside of the scotoma had these animals received a retinal lesion. If analyzed separately, the averages and SDs from these two regions were identical, indicating that there is no labeled pixel density difference between the areas destined to be inside or outside of the scotoma after any treatment. These data also indicate there are no changes in the axon transport of the CTB after AP5 treatment.

The individual axons of the contralateral projection could not be resolved because of the density of label. However, the ventral boundary of this projection remained constant during P0 NMDAR blockade, an indication of little or no expansion of the contralateral axons into the termination zones of the ipsilateral eye or visual cortex.

Although P0 NMDAR blockade did not prevent the refinement 

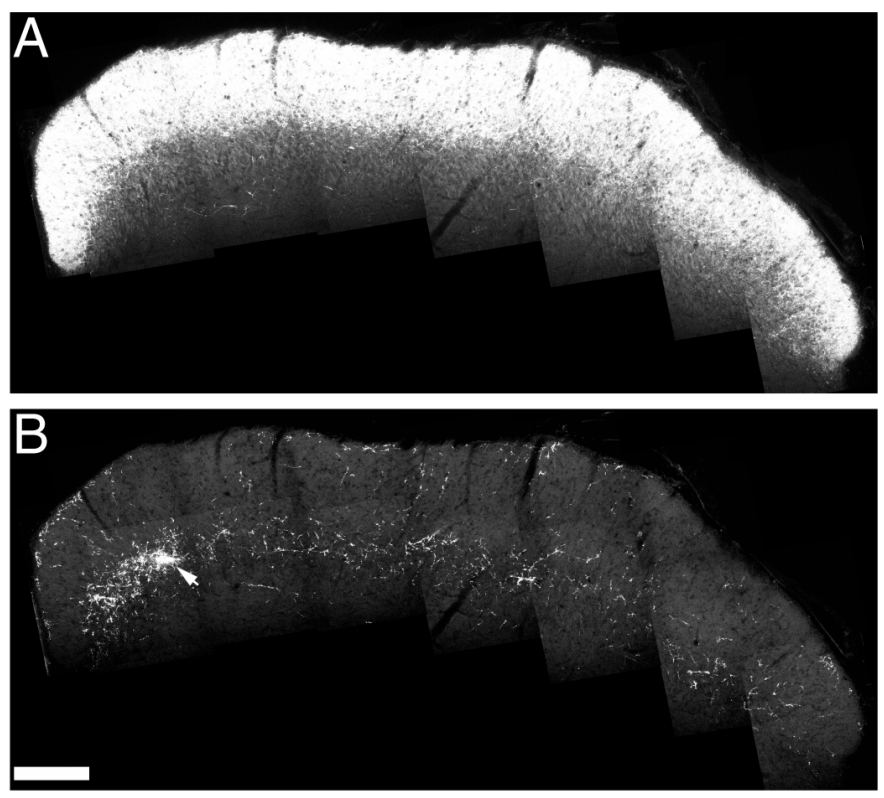

C

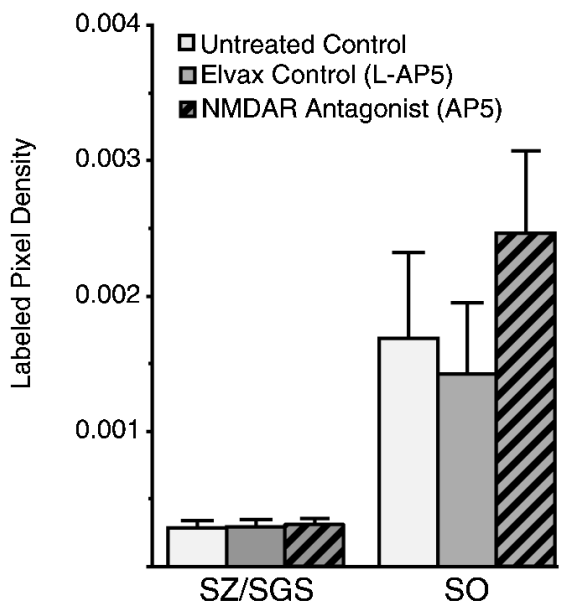

Figure 2. NMDAR blockade from birth does not perturb the refinement of the retinocollicular projection. $A, B$, Montages from a P14 sSC section that has been treated from birth with AP5-Elvax. The section is from a rostrocaudal locale similar to that in Figure 1. Both the contralateral $(A)$ and ipsilateral $(B)$ projections appear normal. In particular, the ipsilateral retinal ganglion cell axons have become normally restricted to the SGS and have refined to form the patchy arborizations (arrow) characteristic of a refined ipsilateral retinal projection. $C$, Quantification of the density of the retinocollicular projection to the ipsilateral sSC from the pups of a single litter that had received either no treatment (control) or chronic treatment with the inactive stereoisomer (L-AP5) or the racemic mixture (AP5) of the competitive NMDA receptor antagonist and were killed at P14. The density of the ipsilateral retinal axon in two-dimensions is represented as the labeled pixel density (see Materials and Methods). These densities were measured in micrographs at least $500 \mu \mathrm{m}$ from the medial or lateral edges using 12 sections taken from three pups of each group. The sections were alternate $100 \mu \mathrm{m}$ sections beginning $400 \mu \mathrm{m}$ from the rostral edge. This sampling pattern was identical to that used to quantify the densities after a lesion. There are no significant differences among any groups (ANOVA). Scale bar: $A, B, 200 \mu \mathrm{m}$.

of the ipsilateral retinocollicular projection at P14, we hypothesized that exposure to AP5 might slow the withdrawal of the projection from the SGS. To address this possibility, two litters were each divided into two groups receiving either L-AP5 or AP5-Elvax at birth. These animals were killed at P6. We observed
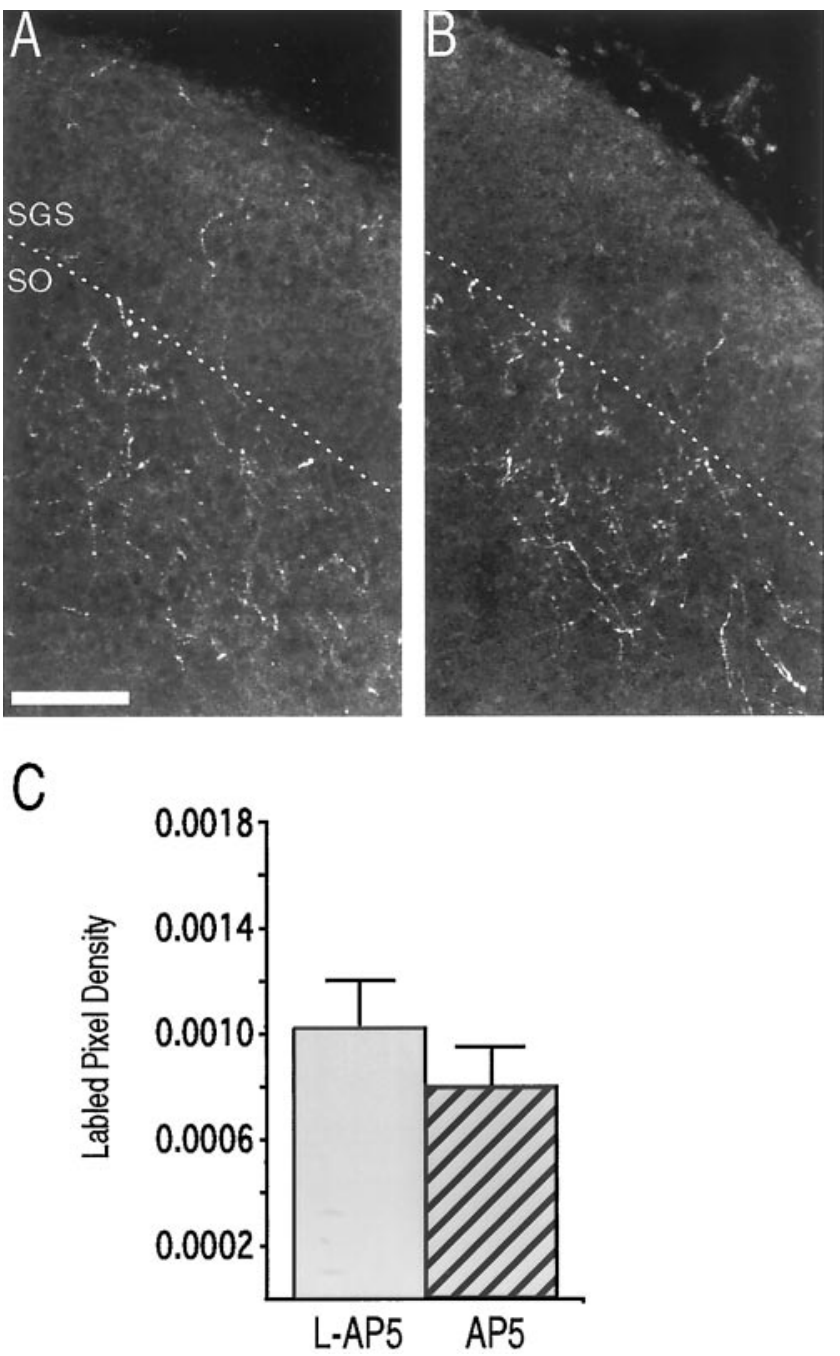

Figure 3. NMDAR blockade does not perturb the refinement of the ipsilateral retinal projection to the sSC at P6. $A, B$, Confocal micrographs from P6 pups chronically treated with L-AP5 (A) or AP5 $(B)$. Only the ipsilateral retinal ganglion cell axons are labeled. The frames are from the lateral half of the rostral sSC. At this early age most of the axons are already confined to the upper SO; few axons extend into the SGS. $C$, Quantification of ipsilateral arbor density in the rostral SGS, at least 300 $\mu \mathrm{m}$ from the medial or lateral edges $(n=18$ sections from 6 pups for the L-AP5 treatment and 15 sections from 5 pups for the AP5 treatment). Scale bar: $A, B, 100 \mu \mathrm{m}$.

that, at P6, like the older pups, most of the ipsilateral projection is restricted to the lower SGS and SO. Chronic blockade of the NMDAR with AP5 did not alter this pattern (Fig. 3A,B). Using a sampling regimen similar to that described above, we observed no difference in the density of ipsilateral axons within the SZ and upper SGS in the rostral sSC (Fig. $3 C)(p=0.36 ; n=18$ sections from 6 L-AP5 and 15 sections from 5 AP5 pups).

\section{Ipsilateral retinocollicular responses to contralateral retinal lesion}

Lesions of the retina produce a retinotopically appropriate zone of deafferentation (a scotoma) in the contralateral sSC and dorsal lateral geniculate nucleus. If these lesions are made during the first few weeks of life, other glutamatergic afferents will sprout to fill some of the vacated space within the scotoma. Both eyes (Lund and Lund, 1971; Frost and Schneider, 1979; Simon et al., 1994), as well as both visual corticies (Mustari and Lund, 1976) 

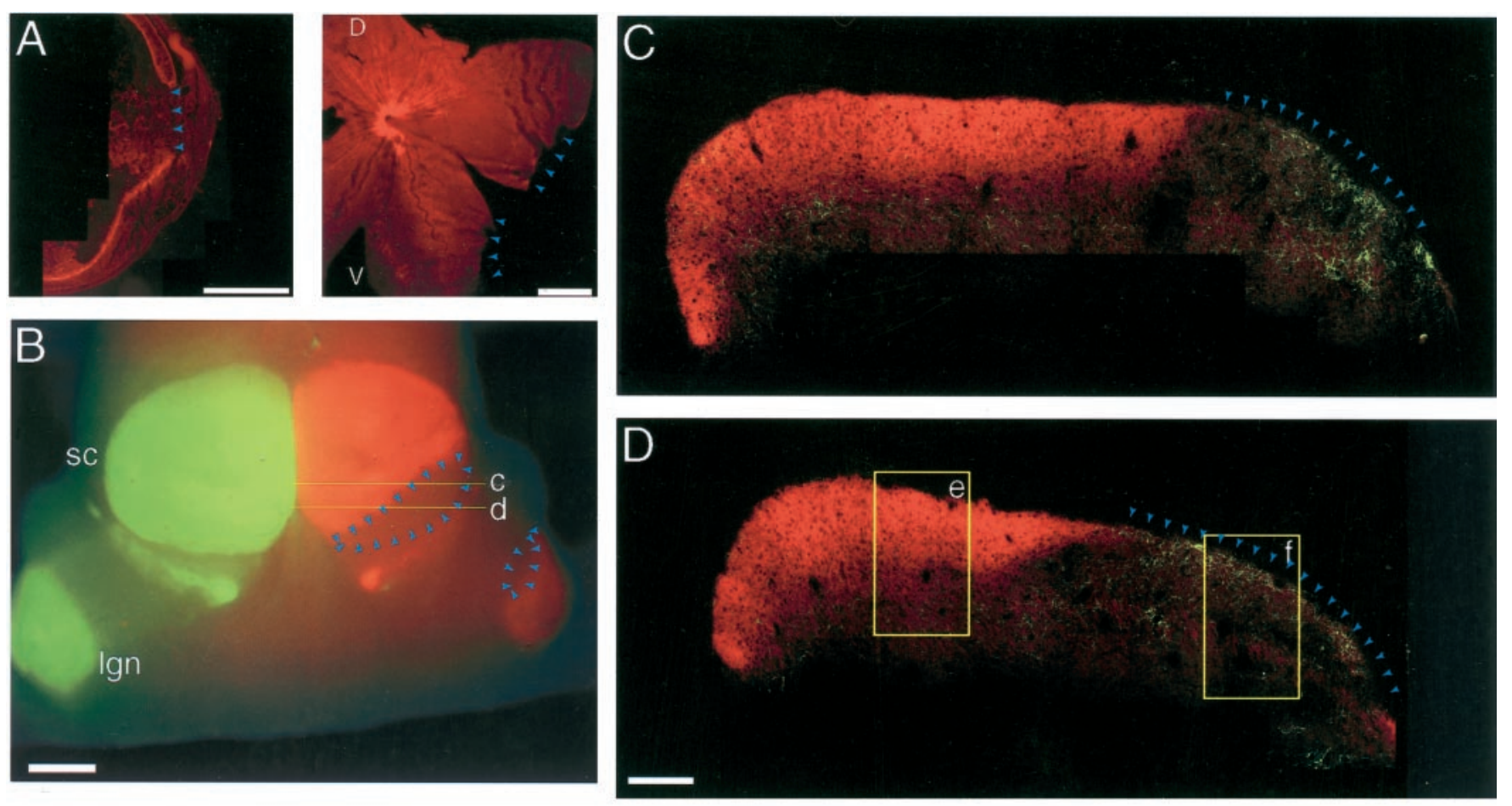

$\mathrm{G}$
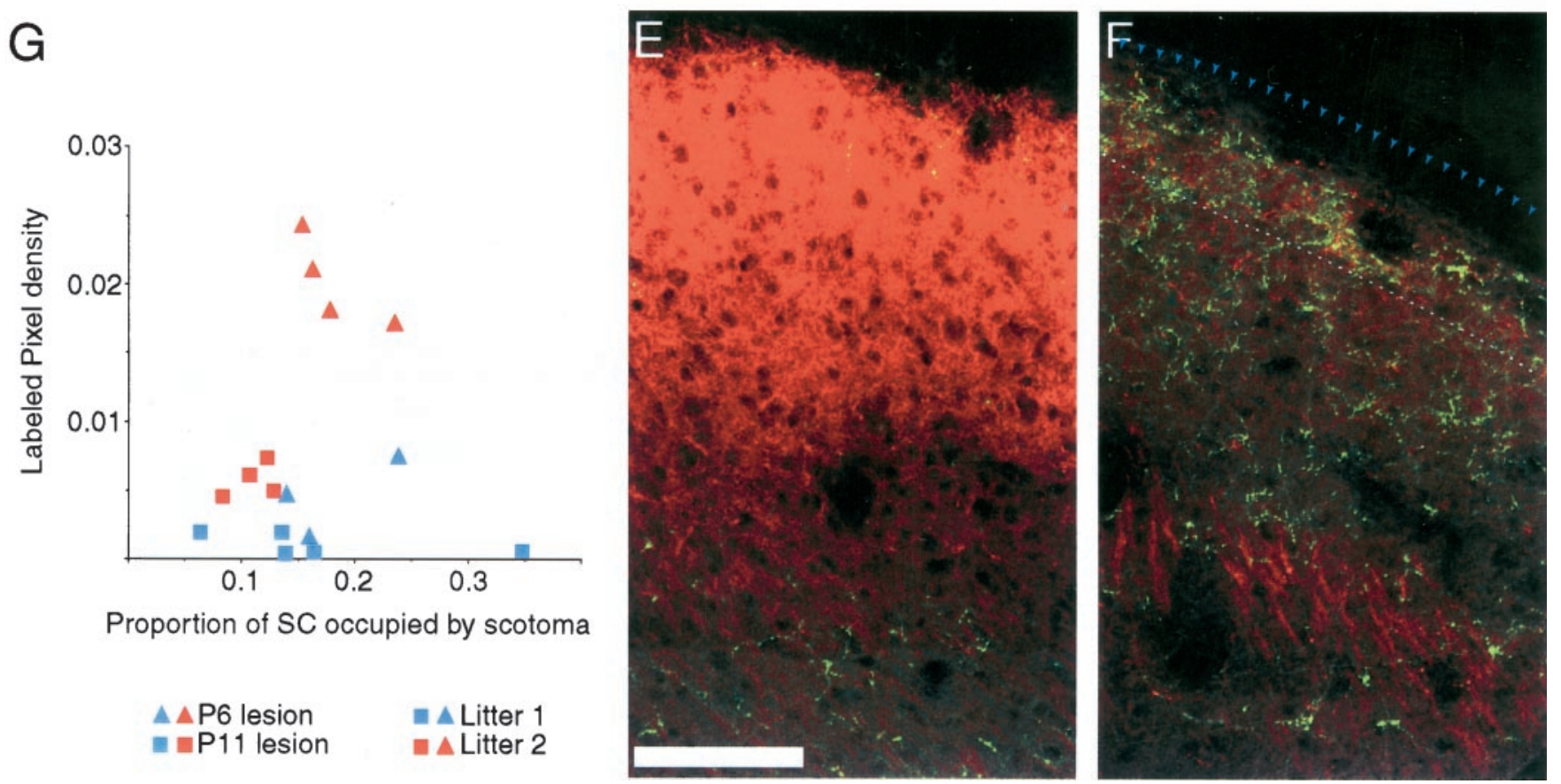

Figure 4. Small retinal lesions made at P6 or P10/P11 cause a scotoma in the contralateral sSC that permits sprouting of the ipsilateral retinal ganglion cell axons. A, Microcautery lesions destroy a small, defined section of retina. Left, The temporal portion of a thin, frontal section of a lesioned retina, $8 \mathrm{~d}$ after the lesion, shows the area of the lesion (arrowheads) with healed sclera surrounding it. The ganglion cell layer is labeled bright red by the CTB. Right, Whole mount of another lesioned retina ( $8 \mathrm{~d}$ after the lesion) shows the ablated area in the temporal pole (arrowheads). There is a reduction in the density of bundled axons entering the optic disk from the lesioned region. $B$, Top view of the dorsal diencephalon and midbrain shows the primary targets of retinal ganglion cells, the sSC (sc) and dorsal lateral geniculate nucleus (lgn). One eye has been labeled with FITC CTB (green) and the other with tRITC CTB (red); this latter eye also received a lesion in the superiotemporal pole of the retina at P6. This lesion causes a scotoma (surrounded by arrowheads) in the laterorostral pole of the contralateral sSC and caudal dorsal LGN 8 d later. $C, D$, Confocal montages are shown of $20 \mu \mathrm{m} z$-series projections taken from coronal sections from the locations marked $c$ and $d$ in $B$. These show sprouting of ipsilateral retinal ganglion cells axons into the SGS and particularly the SZ within the scotoma (arrowheads). E, F, Single frames from the areas shown in $D(e, f)$ illustrate the pattern of sprouting in the SGS and SZ (delineated by the dashed line). $G$, Quantification is shown of ipsilateral axon density within the SGS/SZ inside the scotoma from two litters (one red, the other blue) in which half of the pups received P6 lesions (triangles) and half of the pups received P11 lesions (squares). All animals were killed $8 \mathrm{~d}$ after the lesion. Each point is the average labeled pixel density (see Materials and Methods) for a single animal derived from three sections. Within each litter P6 lesions cause more plasticity than do P11 lesions, but the variability between litters is as large as the difference between early and late lesions within a single litter. Two-way ANOVA indicated significant differences between litters $(p<0.0001)$ and lesion time $(p<0.0001)$. Within litters there is was no relationship between size of the lesion and plasticity. $D$, Dorsal; $V$, ventral. Scale bar: $A, B, 1 \mathrm{~mm} ; C, D, 200 \mu \mathrm{m} ; E, F, 100 \mu \mathrm{m}$. 


\begin{tabular}{|c|c|c|c|c|c|c|c|}
\hline \multirow[b]{2}{*}{ Litter } & & \multicolumn{6}{|l|}{ Treatments } \\
\hline & & $\begin{array}{l}\text { P6 retinal lesion } \\
\left(\times 10^{3}\right)\end{array}$ & $\begin{array}{l}\text { P6 retinal lesion } \\
\text { plus AP5 } \\
\left(\times 10^{3}\right)\end{array}$ & $\begin{array}{l}\mathrm{P} 10 / \mathrm{P} 11 \text { retinal lesion } \\
\left(\times 10^{3}\right)\end{array}$ & $\begin{array}{l}\mathrm{P} 10 / \mathrm{P} 11 \text { retinal } \\
\text { lesion plus } \\
\text { AP5 } \\
\left(\times 10^{3}\right)\end{array}$ & $\begin{array}{l}\mathrm{P} 10 / \mathrm{P} 11 \text { retinal and } \\
\text { cortical lesion } \\
\left(\times 10^{3}\right)\end{array}$ & $\begin{array}{l}\text { P10/P11 retinal and } \\
\text { cortical lesion plus AP5 } \\
\left(\times 10^{3}\right)\end{array}$ \\
\hline \multirow[t]{2}{*}{1} & out & $0.83 \pm 1.01$ & $1.74 \pm 1.58$ & & & & \\
\hline & in & $6.17 \pm 7.47$ & $31.76 \pm 30.93$ & & & & \\
\hline \multirow[t]{2}{*}{2} & out & $0.49 \pm 0.08$ & $0.42 \pm 0.27$ & & & & \\
\hline & in & $7.53 \pm 5.79$ & $21.25 \pm 16.42$ & & & & \\
\hline \multirow[t]{2}{*}{3} & out & & & $0.40 \pm 0.35$ & $0.39 \pm 0.55$ & & \\
\hline & in & & & $16.43 \pm 17.92$ & $26.50 \pm 9.78$ & & \\
\hline \multirow[t]{2}{*}{4} & out & & & $0.14 \pm 0.26$ & $0.63 \pm 0.25$ & & \\
\hline & in & & & $2.07 \pm 2.40$ & $4.19 \pm 5.23$ & & \\
\hline \multirow[t]{2}{*}{5} & out & & & $1.35 \pm 0.54$ & & $2.40 \pm 0.62$ & \\
\hline & in & & & $8.04 \pm 6.05$ & & $42.73 \pm 20.00$ & \\
\hline \multirow[t]{2}{*}{6} & out & & & $0.48 \pm 0.27$ & & $0.98 \pm 0.72$ & \\
\hline & in & & & $5.43 \pm 4.31$ & & $12.41 \pm 5.92$ & \\
\hline \multirow[t]{2}{*}{7} & out & & & & & $1.90 \pm 1.50$ & $2.46 \pm 1.02$ \\
\hline & in & & & & & $23.94 \pm 13.78$ & $59.36 \pm 16.42$ \\
\hline \multirow[t]{2}{*}{8} & out & & & & & $0.78 \pm 0.66$ & $0.78 \pm 0.87$ \\
\hline & in & & & & & $2.01 \pm 1.85$ & $5.96 \pm 6.67$ \\
\hline & out & $0.52 \pm 0.09$ & & $0.45 \pm 0.29$ & & & \\
\hline & in & $4.67 \pm 2.52$ & & $1.00 \pm 0.89$ & & & \\
\hline \multirow[t]{2}{*}{10} & out & $0.27 \pm 0.19$ & & $0.25 \pm 0.28$ & & & \\
\hline & in & $20.00 \pm 3.16$ & & $5.50 \pm 1.29$ & & & \\
\hline
\end{tabular}

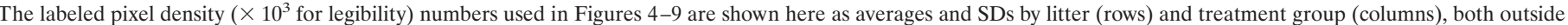

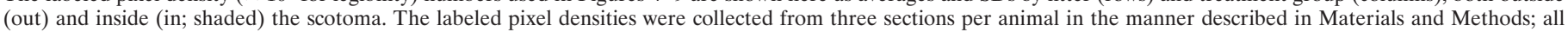
sections from like-treated groups in a single litter were averaged together.

and midbrain afferents (Stevenson and Lund, 1982), are capable of such sprouting. We focused on the response of the ipsilateral and not the contralateral retina to a lesion made in the contralateral eye for two reasons. First, because intraocular injection of fluorescently tagged CTB evenly fills the retinal projection, there is no need to control for injection size. Second, it is difficult to separate refinement from sprouting in the contralateral projection because the latter shows significant exuberance after P0 NMDAR blockade (Simon et al., 1992).

We examined ipsilateral sprouting in response to lesions made at two different times (P6 and P10/P11), with an equal survival time of $8 \mathrm{~d}$ after the lesion. P6 was chosen because it falls within the critical period for this plasticity yet is late enough that the majority of cell death in the retina has already occurred (Crespo et al., 1985; Horsburgh and Sefton, 1987). In addition, by P6 the eye is large enough to make a small lesion reproducibly, and we have demonstrated above that P0 AP5 treatment has no effect on the ipsilateral projection as assayed at P6. We chose P10/P11 because it lies at the end of the critical period for retinal plasticity within the sSC (Lund and Lund, 1976). Furthermore, P11 is the first day that SSC NMDARs have downregulated to produce fast-decaying, adult-like currents (Shi et al., 1997). We originally bracketed this time point by making lesions at P10 or P11 but observed no difference between these groups in the response to any of the lesion conditions we examined; therefore, the two treatments have been collapsed.

The small hole produced by heat lesion of the superior- temporal pole of the retina quickly healed over, but the eye often shrank to reform a smaller sphere. For this reason exact reconstruction of the lesioned area was often difficult, but a gap at the edge of the retina could easily be observed (Fig. 4A), and this produced a consistently placed scotoma in the rostrolateral portion of the superior colliculus (Fig. $4 B$ ). The patterning of the ipsilateral projection outside the region of the scotoma was qualitatively normal. Inside the scotoma, however, an abnormally dense projection from the ipsilateral projection was consistently observed (Fig. $4 C-F$ ). Most of the increased ipsilateral innervation occurs in the SZ, under the pial surface, and in the upper third of the SGS where clumps of arbor can often be observed. All quantitative analysis of the sprouting was measured in the SGS and SZ. The relatively larger and more variant normal projection to the SO makes quantitative analysis of difference in this layer difficult, and it has not been presented. After both P6 and P10/P11 lesions, the amount of sprouting varies greatly, and within the same section we observed areas with little sprouting and others with heavy sprouting (Fig. 4D).

The sprouting was consistently heaviest in the rostral end of the lesion and became lighter caudally. Exact comparison of the ipsilateral axon density between P6 and older pups is difficult because of changes in neuropil size and possibly the axonal transport of CTB. It is obvious, however, that the ipsilateral arbor density within the scotoma (Table 1) is far greater than the normal density at P6 (Fig. 3). This indicates that the response we 

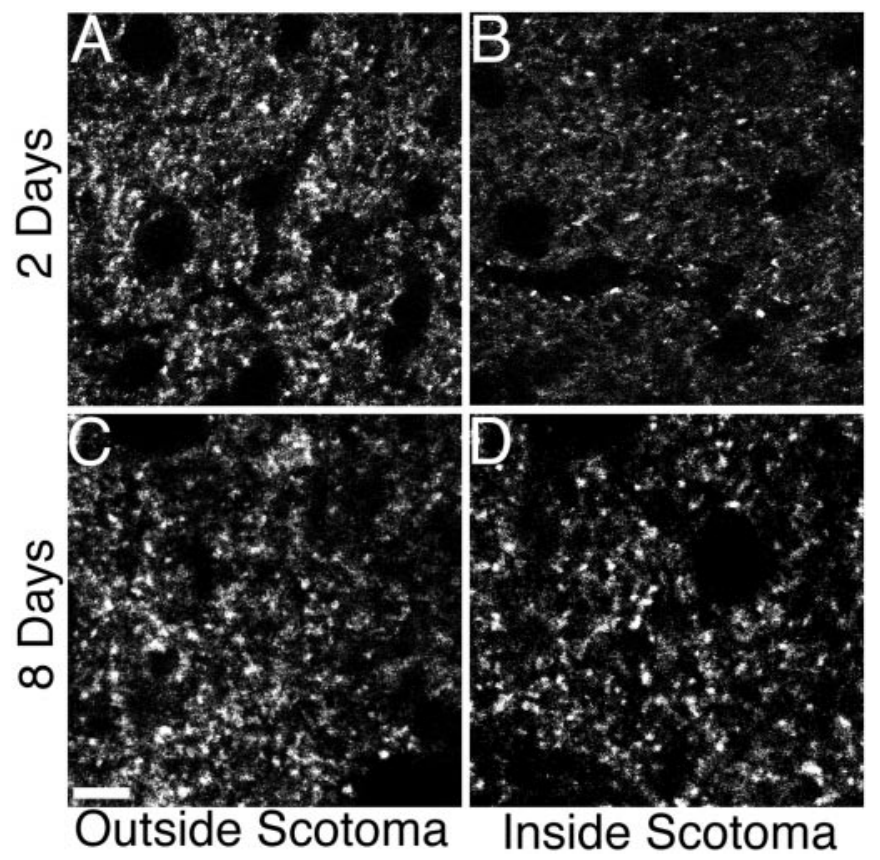

Figure 5. Eight days after the lesion is sufficient to restore normal synaptic density in the SGS. Confocal micrographs of immunohistochemical staining for synaptophysin reveal punctate, presumably synaptic, staining. $A, B$, Two days after a $\mathrm{P} 11$ lesion the puncta are reduced inside the scotoma $(B)$ compared with outside the scotoma $(A)$ in the same section. $C, D$, However, $8 \mathrm{~d}$ after a P11 lesion there is no observable difference inside $(D)$ and outside $(C)$ the scotoma. Scale bar, $10 \mu \mathrm{m}$.

observe is caused by sprouting and not failure of the exuberant ipsilateral arbors to withdraw normally.

Within any single litter, lesions at P6 cause more plasticity than do similar lesions at P11, although the absolute amount of sprouting in the SGS/SZ is considerably different for different litters (Fig. 4G) (two-way ANOVA, by litter, $F=95.02 ; p<0.0001$; by lesion time, $F=76.30 ; p<0.0001)$. There is still significant plasticity after a P10/P11 lesion (see Fig. 8), however, and we have determined that this sprouting is occurring almost entirely after P14 because lesions made at P10 produced no sprouting when the animals were killed $4 \mathrm{~d}$ later (data not shown; $n=6$ animals).

We used synaptophysin immunohistochemistry to examine the effects of contralateral eye lesions on synaptic density. Two days after a P11 retinal lesion, we observed extensive loss of labeled synaptic puncta within the scotoma (Fig. $5 A, B)(n=2)$. However, by $8 \mathrm{~d}$ after the lesion, the synaptic density had qualitatively returned to normal within the scotoma (Fig. 5C,D) $(n=4)$. AP5-Elvax or L-AP5-Elvax treatment had no effect on this recovery of synaptic density (data not shown; $n=3$ each). Although we cannot determine which axons form the new synapses, these data indicate that rapid and extensive synaptic replacement occurs after the lesion. Thus, by the end of the $8 \mathrm{~d}$ survival period when we measure structural plasticity, the synaptic density is relatively normal within the scotoma.

The largest challenge in using the ipsilateral projection as an assay for sprouting was finding a method of controlling for the interanimal and interlitter variability. Because we sampled only from a consistent rostral-caudal region of the lesion, the amount of sprouting was not correlated with the size of the lesion (Fig. $4 G)$. Interanimal sprouting variability was reduced by using the increased labeled pixel density, as outlined in Materials and
Methods, for our statistics. Use of this statistic provides us with a more accurate estimate of the ipsilateral axon density that is caused by new sprouting and not attributable to the natural projection to that region. However, the interlitter variability made it critical to design experiments that divided single litters into two or three groups. In this way we normalized the responses of each litter to the control group and could obtain a relative measure of the effect of a treatment for each litter. In Table 1 we have provided the raw labeled pixel densities for each treatment group by litter that were used in the lesion experiments described below.

\section{Effects of PO NMDAR blockade on sprouting}

To determine whether P0 NMDAR blockade modulated lesioninduced sprouting, we made small retinal lesions on P6 in pups that had been exposed from birth to either L-AP5 or AP5. We observed no difference in the pattern of ipsilateral retinal sprouting after AP5 treatment; the plasticity was still strictly confined within the scotoma, and most of the sprouting occurred in the SZ and upper SGS (Fig. $6 E$ vs $D$ ). However, the amount of sprouting was significantly greater after AP5 treatment. The additional density was not added evenly throughout the scotoma, however, but occurred in large, dense patches, most heavily along the rostral region of the sSC. In particular, a small, dense cluster usually formed along the far lateral edge of the SGS. To quantify the increased sprouting, increased labeled pixel densities were obtained from the SGS/SZ of pups from two litters, half of each treated with L-AP5 and the other half treated with AP5 ( $n=24$ sections from 8 animals in each group). This analysis revealed a fourfold increase in sprouting within the center of the lesion after AP5 treatment (Fig. 7) $(p<0.01)$ compared with the L-AP5 control. Because accurately identifying the SGS and SO is difficult at the lateral edge where ipsilateral innervation was generally greatest, we did not measure labeled pixel density in this area, and so our measure of increased sprouting underestimates the total additional sprouting after AP5 treatment.

P0 NMDAR blockade from birth did not cause a similar enhancement of ipsilateral retinocollicular sprouting after a P10/ P11 retinal lesion (Fig. $8 A$ ). The increased labeled pixel densities gathered from the SGS/SZ of two litters, divided, and quantified as described above $(n=21$ sections from 7 L-AP5-treated pups and 15 sections from 5 AP5-treated pups) showed no significant difference in increased axon density within the scotoma (Fig. $8 A_{5}$ ).

\section{Occipital cortex lesions restore the plasticity enhancement of PO NMDAR blockade after late eye lesions}

Why did NMDAR blockade increase plasticity to a P6 lesion but not one made at P10/P11 although there was substantial sprouting in both cases? One possibility is that between these two stages another converging projection became a more effective competitor for the sSC space vacated by the contralateral projection. The primary candidate for such a competitor is the ipsilateral visual cortex, which sends a dense, visuotopic projection that arborizes in the mid and lower SGS (for review, see Huerta and Harting, 1984). The elaboration of cortical arbors into the SGS does not begin until after P7 (Lopez-Medina et al., 1989), and the refinement of the cortical projections is still occurring late into the second postnatal week (Thong and Dreher, 1986; Lopez-Medina et al., 1989; Binns and Salt, 1997).

To examine whether the visual cortex is a late competitor of the ipsilateral retina, in half the pups of each of two litters that received no Elvax implantation, the occipital cortex was aspirated 

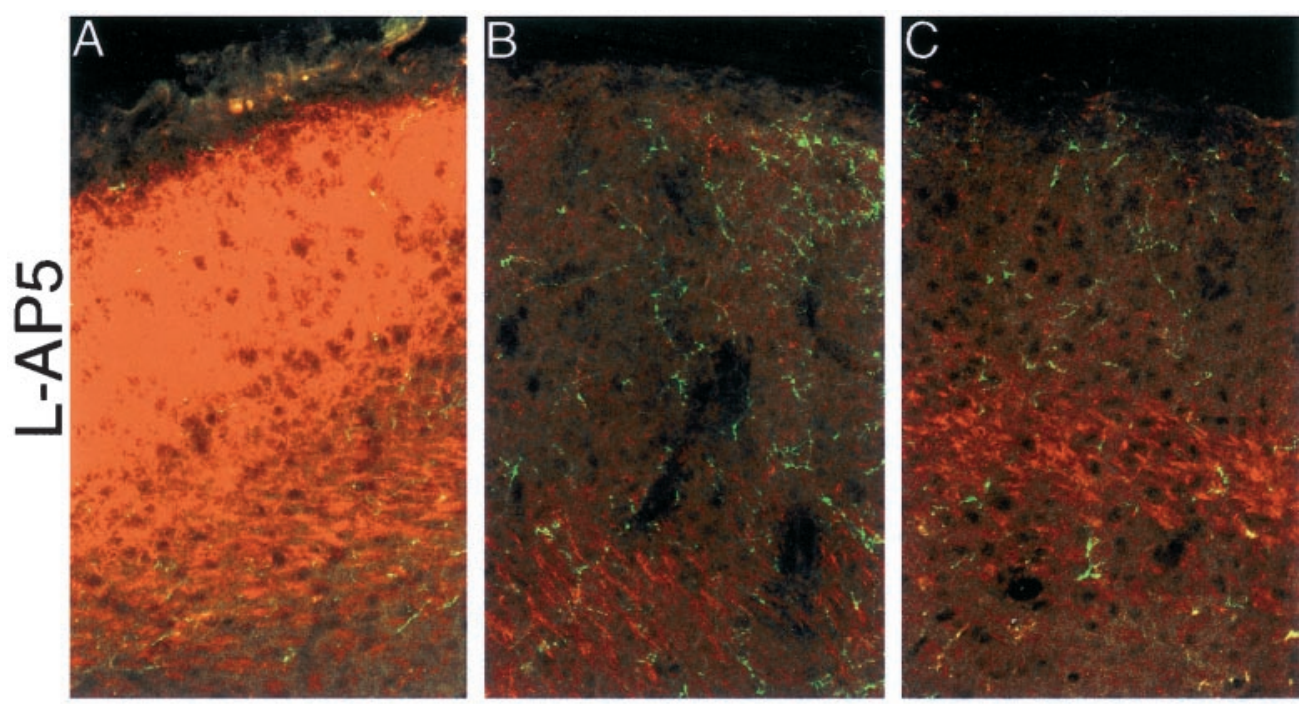

Figure 6. NMDA receptor blockade from birth increases the sprouting in response to a P6 retinal lesion. Confocal micrographs of a $20 \mu \mathrm{m} z$-series projection show a greater density of ipsilateral retinal ganglion cell axons within $(B, C$, $E, F)$, but not outside $(A, D)$, the scotoma when sections from pups treated with L-AP5 $(A-C)$ are compared with those treated with AP5 $(D-E) . B$ and $E$ show a characteristic response in the crown of the rostral sSC, whereas $C$ and $F$ show a more lateral region. Scale bar, $100 \mu \mathrm{m}$.
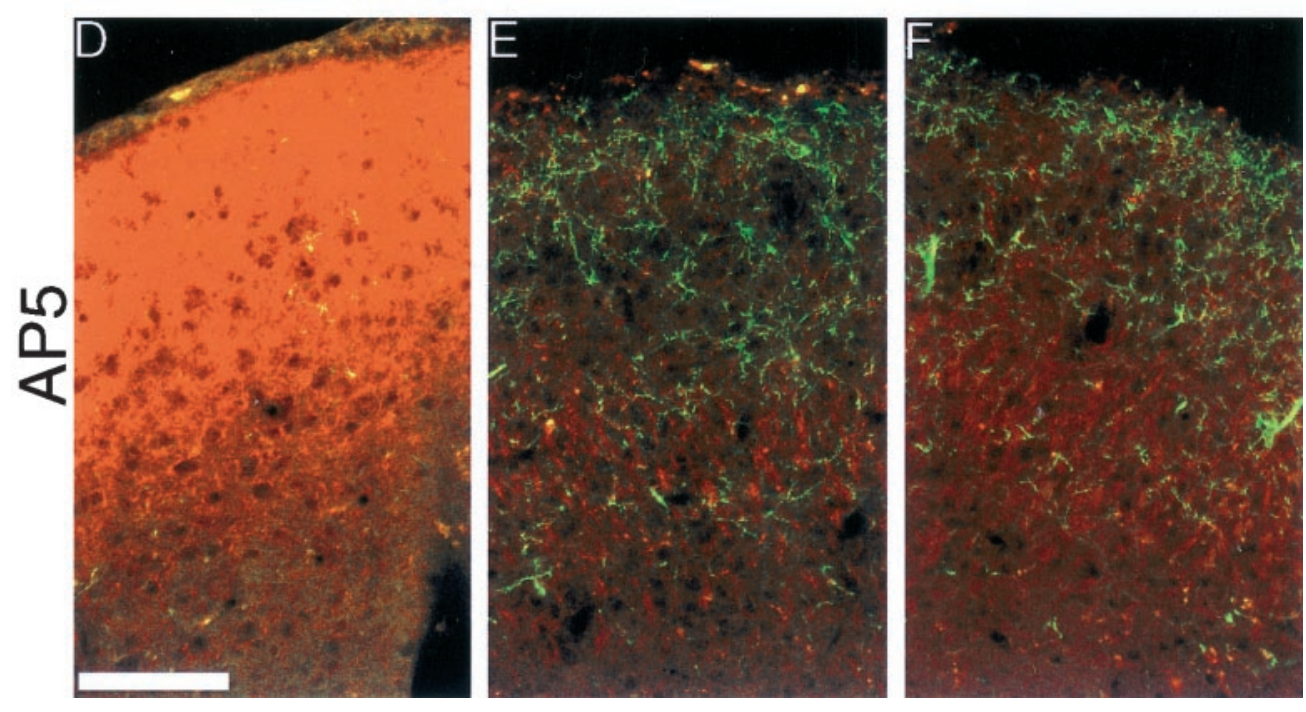

at P10/P11 simultaneous with a contralateral retinal lesion in all the pups. Cortical lesions caused an average $12.4 \%$ decrease in the surface area of the sSC $(p<0.01)$ but no noticeable decrease in the thickness of the SGS. In addition, cortex removal significantly increased the plasticity to a $\mathrm{P} 10 / \mathrm{P} 11$ retinal lesion (Fig. $8 B$ ). The pattern of the additional arborization within the scotoma was widely divergent, most often occurring in the SZ (Fig. $8 B_{4}$ ), but extensive sprouting in the mid and lower SGS was also observed (Fig. $8 C_{2}$ ). The increased labeled pixel density within the SGS/SZ after a P10/P11 occipital cortex and contralateral retinal lesion was almost threefold that observed for a retinal lesion alone (Fig. $\left.8 B_{5}\right)(n=21$ sections from 7 animals for the retinal lesion alone and 24 sections from 8 animals for the combined lesion; $p<0.01$ ), greatly above the increased density that would be expected to result from the shrinkage of the sSC alone.

We next explored the effect of P0 NMDAR blockade on ipsilateral sprouting after combined retinal and cortical lesions at P11 (Fig. 8C). Two litters were each divided into two groups that received L-AP5 or AP5 Elvax at birth; all pups then underwent combined cortical and retinal lesion at P11. With the occipital cortex removed, P0 AP5 treatment increased the sprouting after the retinal lesion. This is the effect that AP5-Elvax has after P6 retinal lesion but with the visual cortex intact. For the AP5 treatment group, the increased labeled pixel density was almost threefold that of the increased labeled pixel density of L-AP5treated littermates (Fig. $\left.8 C_{5}\right)(n=18$ sections from 6 animals for each group; $p<0.05)$. We observed extensive variability in the pattern and amount of sprouting. A large portion of the sprouting occurred in the SZ and upper SGS as usual. However, in some pups much new arborization also accrued in the lower SGS.

\section{Effect of cortical lesion outside the scotoma}

If NMDAR blockade operates to increase ipsilateral retinal sprouting only under conditions in which the ipsilateral projection is a dominant competitor, AP5 treatment should not increase any ipsilateral plasticity caused by lesion of the visual cortex alone because the more dense contralateral retinal projection would still be the dominant competitor. Thus, in our experiments, outside the scotoma, where the contralateral retinal projection is intact, we should not observe any extra sprouting caused by AP5 treatment over that induced by the cortical lesion alone. To examine this possibility we used the ipsilateral retinal axon density measured in micrographs taken outside the scotoma, where the contralateral retina was intact. The pups used in this analysis were drawn from a subset of the above experiments. All of these pups were P19 at death and were imaged under identical conditions. The labeled pixel densities in the SGS/SZ outside the lesion were statistically similar for similar treatment groups across different 


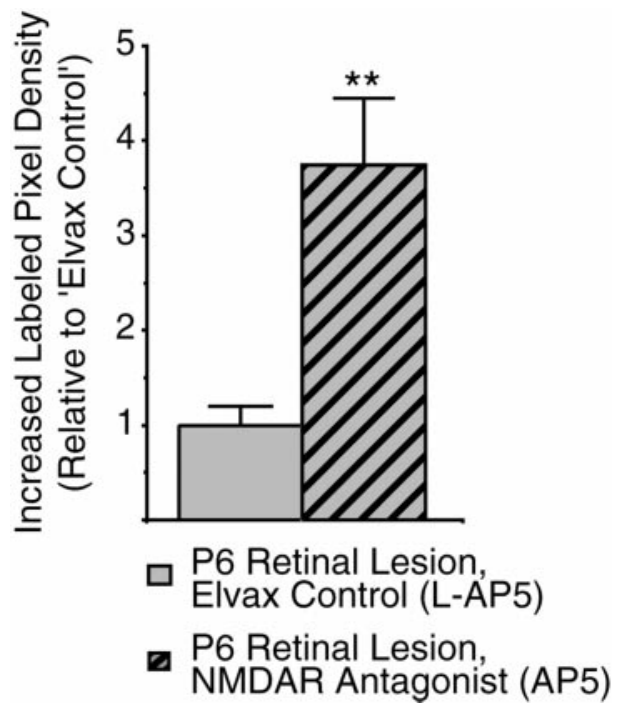

Figure 7. Quantification of the increased ipsilateral retinal axon sprouting in the SGS/SZ in response to a P6 retinal lesion after chronic NMDAR blockade. The amount of sprouting into the scotoma is measured in each section by subtracting the labeled pixel density (see Materials and Methods) of a frame outside the scotoma from that of a frame inside the scotoma. To control interlitter variability, this increased labeled pixel density is normalized to the average response of the L-AP5 pups from each litter. The data were gathered from two litters, each divided into L-AP5-Elvax- and AP5-Elvax-treated groups. From each pup increased labeled pixel densities were measured from three sections in the rostral region of the scotoma (see Materials and Methods). Double asterisks indicate $p<0.01$ by $t$ test.

litters, so the densities were not normalized. There was also no difference between L-AP5-treated pups and pups that received no Elvax. Consequently, data from these animals were pooled. The data from single, intralitter, comparisons were identical to the pooled data. A two-way ANOVA indicated that, as predicted, occipital cortex lesions induced plasticity outside the scotoma, and AP5 treatment from birth did not increase this ipsilateral projection plasticity further, although this same treatment increased ipsilateral sprouting within the scotoma (Fig. 9) (three sections from each pup; P11 retinal lesion alone, $n=30$; P11 retinal lesions and P0 AP5 treatment, $n=15$; P11 retinal and cortical lesions, $n=18$; P11 retinal and cortical lesions plus P0 AP5 treatment, $n=18 ; F=15.46, p<0.001$ for effect of cortical lesion; $F=0.06, p=0.81$ for effect of AP5 treatment).

\section{DISCUSSION}

This study has used the high-sensitivity, anterograde tracer CTB (Angelucci et al., 1996) to study sprouting of the ipsilateral retinocollicular projection of the rat. We have quantitatively shown that there is an appreciable downregulation of sprouting between P6 and P10/P11 but that substantial plasticity is possible after late lesions. We have shown that much of this plasticity loss is caused by competition between converging retinal and central visual inputs. Furthermore, we have demonstrated that early and chronic perturbation of NMDAR function increases ipsilateral sprouting via a mechanism that is independent of the role of the receptor in mediating the activity-dependent phases of synaptic competition.

Before discussing these data it is important to mention that we have used a quantitative and relatively high-resolution approach in these analyses to discriminate between the multiple factors that can impact the ipsilateral retinal sprouting response, for example, competitive afferents, growth inhibitors in myelin, and adhesion molecules. This has proven to be an effective approach to separate at least two factors, competition and NMDAR activity. To accomplish this analysis, we have developed protocols that overcome a number of technical difficulties inherent in most CNS studies of sprouting. First, there is tremendous variability in the sprouting response to a perturbation. We incorporated several procedures that minimized this complication. For example, instead of enucleation, we used small lesions of the contralateral retina. By leaving most of the contralateral retinal projection intact, secondary degeneration of central visual regions was avoided; we saw no sSC shrinkage within the scotoma. The small lesion also allowed us to estimate the actual sprouting of the ipsilateral projection within the scotoma by comparing it with ipsilateral axon density outside of the scotoma at the same rostrocaudal level, which reduced interanimal variability. We also carefully restricted our analyses of ipsilateral axon density to the same region of rostral $\mathrm{sSC}$, thereby removing the variability introduced by normal differences in the arborization pattern of the ipsilateral projection across the sSC. Most critically, we focused all comparisons on pups from within the same litter. This reduced a very large interlitter variability in the degree of sprouting. A second major problem with studies of sprouting is the difficulty in making quantitative measurements of the sprouting response. The availability of confocal microscopy and of robust, bright, anterograde labels for the retinal projections has aided us in this regard by allowing separation of signal and background fluorescence. Thus, we were able to create binary images with a threshold low enough to include all in-focus axons and arbors and to exclude reliably everything else.

It has long been clear that both the contralateral retina and the ipsilateral cortex are potential competitors of the ipsilateral retinal axons for synaptic space (Lund and Lund, 1971; Rhoades et al., 1982). Developmental studies of the ipsilateral projection indicated that much of the plasticity observed reflected a failure to retract an initially exuberant projection (Land and Lund, 1979). There is also evidence that a relevant factor in the normal retraction of ipsilateral axons is, in part, the activity and not just the physical presence of the competing projections (Fawcett et al., 1984). Furthermore, action potential activity in the eye is critical for proper refinement of the ipsilateral projection (Thompson and Holt, 1989). The current results provide several important amendments and qualifications to these previous observations. We find, using the CTB-tracing technique, that retinal lesions as late as P11 can induce a robust ipsilateral sprouting into the scotoma by P19. Our results are similar to those of Bastos et al. (1999), who show plasticity when late lesions are followed by a 1 week survival. We have demonstrated that the projection has retracted from its early exuberance by P6, and thus the plasticity to even relatively early lesions is a result of sprouting, not just a failure of existing axons to withdraw. Our data also provide two indications that the corticocollicular projection is an important downregulator of ipsilateral retinal sprouting in the sSC, even before eye opening (P13-P14). First, a within-litter comparison of the degree of ipsilateral sprouting into the scotoma caused by P6 versus P10/P11 lesions indicated significantly more robust sprouting with the earlier lesion. Second, NMDAR blockade from birth fails to increase ipsilateral retinal sprouting induced by a P10/P11 lesion, although the same treatment is effective at increasing sprouting after the P6 lesion. Removal of the ipsilateral cortex allows increased ipsilateral retinal sprouting after P0 NMDAR blockade from birth, indicating that suppression by the cortical 

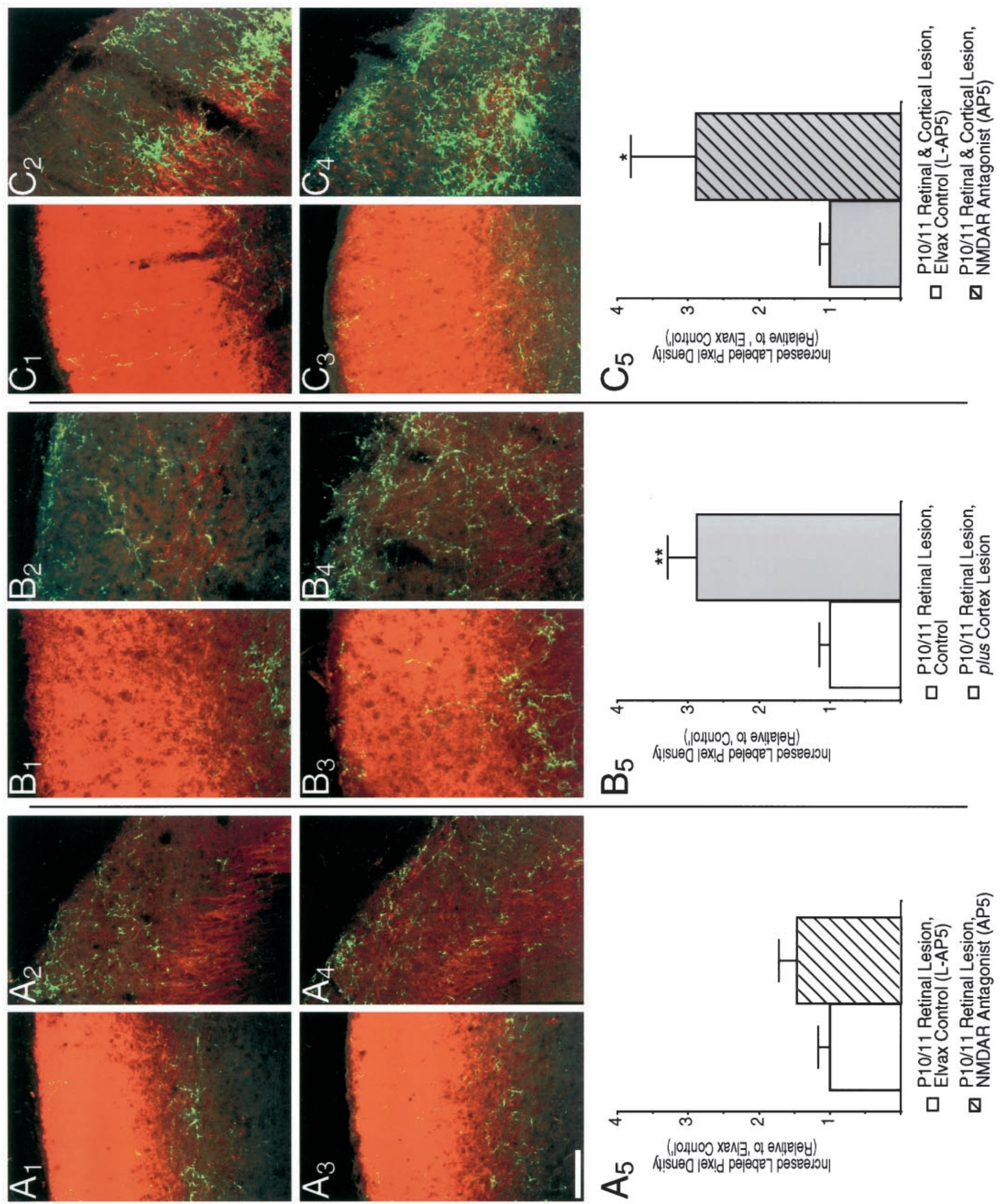

Figure 8. The occipital cortex inhibits ipsilateral retinal sprouting in response to a P10/P11 retinal lesion, and its removal allows NMDAR blockade to augment this sprouting. $A$, NMDAR blockade from birth does not affect the sprouting in response to a P10 retinal lesion. $A_{1}-A_{4}$, Confocal micrographs of a $20 \mu \mathrm{m} z$-series projection show that both L-AP5 $\left(A_{1}, A_{2}\right)$ - and D-AP5 $\left(A_{3}, A_{4}\right)$-treated pups have similar ipsilateral retinal axon densities within $\left(A_{2}\right.$, $\left.A_{4}\right)$ and outside $\left(A_{1}, A_{3}\right)$ the scotoma. $A_{5}$, Quantification is as described in Figure 7. B, Removal of the ipsilateral occipital cortex (Figure legend continues) 


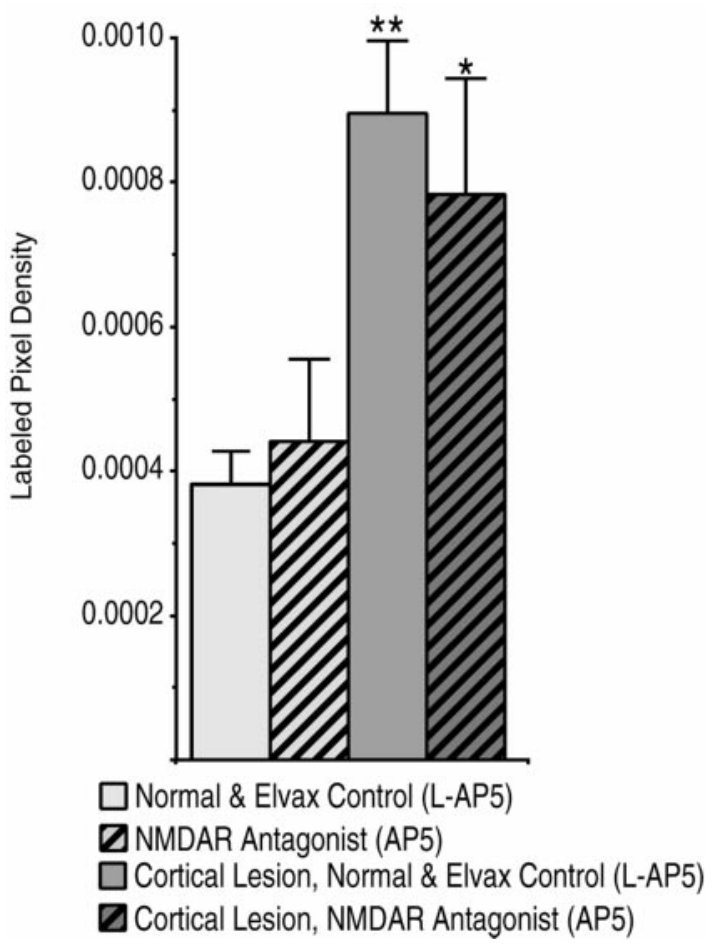

Figure 9. Ipsilateral retinal ganglion cell axon density outside the scotoma is increased by removal of the occipital cortex but not by NMDA receptor blockade from birth (Fig. 8, compare odd-numbered frames). Labeled pixel intensities were calculated for three frames outside the scotoma (Fig. $4 D$, box $e$ ) from three sections of each pup from the litters used in the previous experiments. Normal and L-AP5-Elvax treatment groups showed no differences and were grouped for this comparison. Single and double asterisks represent $p<0.05$ and $p<0.01$ differences from control, respectively, by Tukey pairwise post hoc comparison.

projection was a relevant parameter inhibiting sprouting at P10/ $\mathrm{P} 11$ but not at P6. These age-dependent observations make sense in terms of a competing, corticocollicular projection that follows its own timetable of differentiation regardless of the contralateral retinal lesion. In normal rats the corticocollicular projection is present in the colliculus in the neonate, but it does not begin to arborize within the sSC until the second postnatal week. This refinement is not complete until well into the third postnatal week (Lopez-Medina et al., 1989).

One of our motivations in undertaking this study was to examine the role played by the NMDAR in the competitive interactions that occur among the converging visual projections within the sSC. The current work has been unambiguous in this regard. Blockade of the NMDAR from P0 does not disrupt the competition between retinal afferents in the SSC that causes the more robust, or "normal," projections to these laminae to suppress sprouting of the ipsilateral retinal input. The P0 NMDAR blockade also does not cause the contralateral retinal input to invade the SO, the normal terminal area of the ipsilateral projection. The clustering of the ipsilateral projection in the SO is also not disrupted by the early-onset AP5 treatment. All of these visual projections are glutamatergic (Huerta and Harting, 1984) and use NMDA receptors to transmit part of their activity (Hestrin, 1992; Binns and Salt, 1998) as early as P6 (Shi et al., 2001). Furthermore, much refinement of the ipsilateral projection is dependent on nitric oxide (Vercelli et al., 2000), often thought to be downstream of NMDAR activation in the developmental refinements of some projections (Hahm et al., 1991; Cramer et al., 1996; Ernst et al., 1999). Increasing serotonin levels causes sprouting of ipsilateral retinal terminals (Mooney et al., 1998; Bastos et al., 1999) and increases lesion-induced sprouting (Bastos et al., 1999). However, our results indicate that the activity needed to suppress sprouting and refine the ipsilateral projection need not come via the NMDAR. It may instead be caused by AMPA and/or metabotropic channel activation or the significant retinally driven cholinergic input to ipsilateral patches that arises via the parabigeminal nucleus (Stevenson and Lund, 1982; Huerta and Harting, 1984).

Nevertheless, we have found that early blockade of the NMDAR does increase plasticity although competition appears to be intact. We suggest that the dominant factor in the results we have obtained is that our early blockade has a novel and primarily nonsynaptic effect on the sSC neuropil and, specifically, that the early NMDAR blockade has retarded the development of a number of currently unknown factors in the sSC, thereby rendering the neuropil more permissive to sprouting by all projections. This hypothesis is supported by studies showing that two molecular indices of synaptic maturation do not occur in sSC neuropil blocked from birth with AP5, namely, the normal increase in NMDAR1 mRNA (Hofer et al., 1994) and CaM kinase II activity (Scheetz et al., 1996), and also by an anatomical study of the refinement of the contralateral retinal projection after P0 NMDAR blockade (Simon et al., 1992). The latter study revealed maintenance of exuberant, topographically inappropriate contralateral retinal projections within the sSC at least 1 week after they were withdrawn in normal animals. This result was interpreted as evidence of a failure to withdraw synapses with poorly correlated activity. However, it could equally reflect profuse sprouting of the contralateral retinal input after early NMDAR blockade. In this context it is not particularly unexpected that the less dense ipsilateral projection does not sprout after P0 NMDAR blockade with a denser competing input, except when that input is removed. A similar interaction accounts for the failure of the ipsilateral projection to further sprout into the scotoma produced by a P10/P11 lesion after P0 NMDAR blockade. In these later experiments the normal arborization of the

\section{$\longleftarrow$}

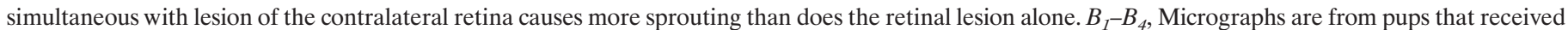

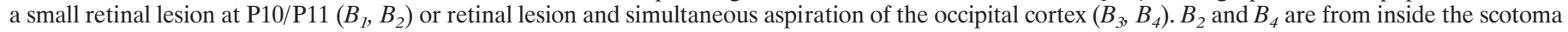

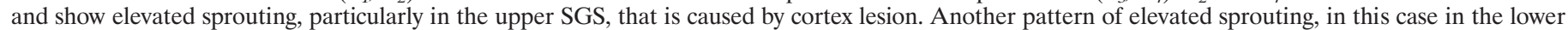

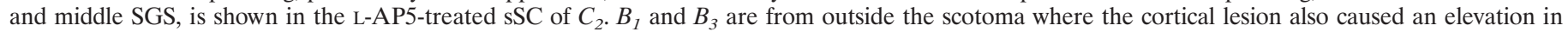

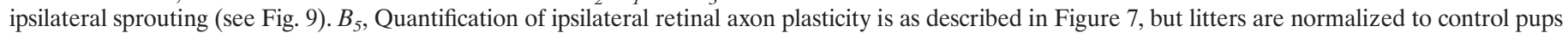

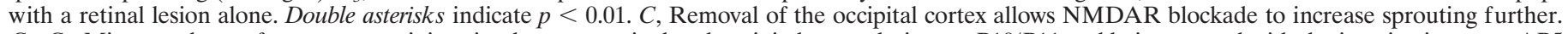

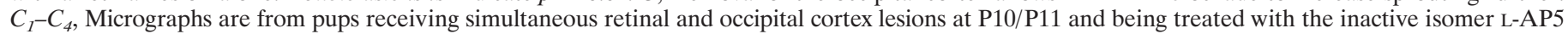

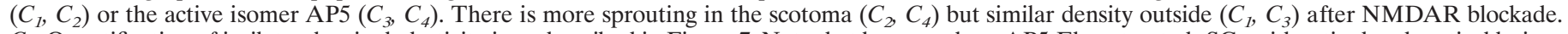

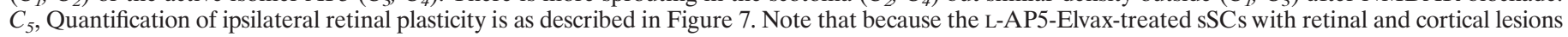

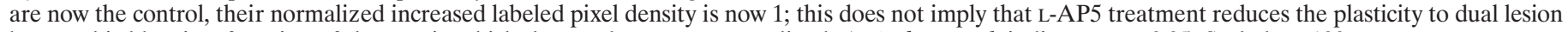
by one-third but is a function of the way in which the numbers were normalized. A single asterisk indicates $p<0.05$. Scale bar, $100 \mu \mathrm{m}$. 
corticocollicular projection, which occurs during the third postnatal week, would be expected to be amplified, and so the denser cortical input would outcompete the ipsilateral input.

In short, our data indicate a pronounced sprouting ability of the ipsilateral retinal projection and demonstrate competitive interactions between this input and both the contralateral retinal and ipsilateral corticocollicular input. They also suggest that early NMDAR blockade generally facilitates sprouting in the sSC neuropil, without eliminating an innervation density-dependent competition. However, because we do not yet know how nonNMDA glutamate receptors have regulated their function in response to this early-onset chronic blockade, we cannot rule out that compensatory changes have allowed synaptic competition to continue, despite the blockade of NMDARs.

\section{REFERENCES}

Angelucci A, Clasca F, Sur M (1996) Anterograde axonal tracing with the subunit B of cholera toxin: a highly sensitive immunohistochemical protocol for revealing fine axonal morphology in adult and neonatal brains. J Neurosci Methods 65:101-112

Bandtlow CE, Schwab ME (2000) NI-35/250/nogo-a: a neurite growth inhibitor restricting structural plasticity and regeneration of nerve fibers in the adult vertebrate CNS. Glia 29:175-181.

Bastos EF, Marcelino JL, Amaral AR, Serfaty CA (1999) Fluoxetineinduced plasticity in the rodent visual system. Brain Res 824:28-35.

Binns KE, Salt TE (1997) Post eye-opening maturation of visual receptive field diameters in the superior colliculus of normal- and darkreared rats. Dev Brain Res 99:263-266.

Binns KE, Salt TE (1998) Developmental changes in NMDA receptormediated visual activity in the rat superior colliculus, and the effect of dark rearing. Exp Brain Res 120:335-344.

Cline HT, Constantine-Paton M (1989) NMDA receptor antagonists disrupt the retinotectal topographic map. Neuron 3:413-426.

Cline HT, Debski EA, Constantine-Paton M (1987) $N$-methyl-Daspartate receptor antagonist desegregates eye-specific stripes. Proc Natl Acad Sci USA 84:4342-4345.

Cramer KS, Angelucci A, Hahm JO, Bogdanov MB, Sur M (1996) A role for nitric oxide in the development of the ferret retinogeniculate projection. J Neurosci 16:7995-8004.

Crespo D, O'Leary DDM, Cowan WM (1985) Changes in the numbers of optic nerve fibers during late prenatal and postnatal development in the albino rat. Brain Res 351:129-134.

Daw NW (1995) Visual development. New York: Plenum.

Ernst AF, Wu HH, El-Fakahany EE, McLoon SC (1999) NMDA receptor-mediated refinement of a transient retinotectal projection during development requires nitric oxide. J Neurosci 19:229-235.

Fawcett JW, O'Leary DDM, Cowan WM (1984) Activity and the control of ganglion cell death in the rat retina. Proc Natl Acad Sci USA 81:5589-5593.

Frost DO, Schneider GE (1979) Plasticity of retinofugal projections after partial lesions of the retina in newborn Syrian hamsters. J Comp Neurol 185:517-567.

Garraghty PE, Muja N (1996) NMDA receptors and plasticity in adult primate somatosensory cortex. J Comp Neurol 294:319-326.

Hahm JO, Langdon RB, Sur M (1991) Disruption of retinogeniculate afferent segregation by antagonists to NMDA receptors. Nature $351: 568-570$

Hestrin S (1992) Developmental regulation of NMDA receptormediated synaptic currents at a central synapse. Nature 357:686-689.

Hofer M, Prusky GT, Constantine-Paton M (1994) Regulation of NMDA receptor mRNA during visual map formation and after receptor blockade. J Neurochem 62:2300-2307.

Horsburgh GM, Sefton AJ (1987) Cellular degeneration and synaptogenesis in the developing retina of the rat. J Comp Neurol 263:553-566.

Huerta MF, Harting JK (1984) The mammalian superior colliculus: studies of its morphology and connections. In: Comparative neurology of the optic tectum (Vanegas H, ed) pp 687-818. New York: Plenum.

Kirkwood A, Lee HK, Bear MF (1995) Co-regulation of long-term potentiation and experience-dependent synaptic plasticity in visual cortex by age and experience. Nature 375:328-331.

Kleinschmidt A, Bear MF, Singer W (1987) Blockade of "NMDA" receptors disrupts experience-dependent plasticity of kitten striate cortex. Science 238:355-358.

Land PW, Lund RD (1979) Development of the rat's uncrossed retinotectal pathway and its relation to plasticity studies. Science 205:698-700

Lopez-Medina A, Bueno-Lopez JL, Reblet C (1989) Postnatal development of the occipito-tectal pathway in the rat. Int $\mathrm{J}$ Dev Biol 33:277-286.

Lund RD (1978) Development and plasticity of the brain. New York: Oxford UP.

Lund RD, Lund JS (1971) Synaptic adjustment after deafferentation of the superior colliculus of the rat. Science 171:804-807.

Lund RD, Lund JS (1976) Plasticity in the developing visual system: the effects of retinal lesions made in young rats. J Comp Neurol 169:133-154.

Lund RD, Cunningham TJ, Lund JS (1973) Modified optic projections after unilateral eye removal in young rats. Brain Behav Evol 8:51-72.

Mooney RD, Crnko-Hoppenjans TA, Ke M, Bennett-Clarke CA, Lane RD, Chiaia NL, Rhoades RW (1998) Augmentation of serotonin in the developing superior colliculus alters the normal development of the uncrossed retinotectal projection. J Comp Neurol 393:84-92.

Mustari MJ, Lund RD (1976) An aberrant crossed visual corticotectal pathway in albino rats. Brain Res 112:37-44.

Prusky GT, Ramoa AS (1999) Novel method of chronically blocking retinal activity. J Neurosci Methods 87:105-110.

Rhoades RW, Kuo DC, Polcer JD (1982) Effects of neonatal cortical lesions upon retinocollicular projections in the hamster. Neuroscience 7:2441-2458.

Scheetz AJ, Prusky GT, Constantine-Paton M (1996) Chronic NMDA receptor antagonism during retinotopic map formation depresses $\mathrm{CaM}$ kinase II differentiation in rat superior colliculus. Eur J Neurosci 8:1322-1328.

Schlaggar BL, Fox K, O'Leary DD (1993) Postsynaptic control of plasticity in developing somatosensory cortex. Nature 364:623-626.

Shi J, Aamodt SM, Constantine-Paton M (1997) Temporal correlations between functional and molecular changes in NMDA receptors and GABA neurotransmission in the superior colliculus. J Neurosci 17:6264-6276

Shi J, Townsend MT, Constantine-Paton M (2000) Activity-dependent induction of tonic calcineurin activity mediates a rapid developmental down-regulation of NMDA receptor currents. Neuron 28:103-114.

Simon DK, Prusky GT, O’Leary DDM, Constantine-Paton M (1992) $\mathrm{N}$-methyl-D-aspartate receptor antagonists disrupt the formation of a mammalian neural map. Proc Natl Acad Sci USA 89:10593-10597.

Simon DK, Roskies AL, O'Leary DD (1994) Plasticity in the development of topographic order in the mammalian retinocollicular projection. Dev Biol 162:384-393.

Smith AL, Cordery PM, Thompson ID (1995) Manufacture and release characteristics of Elvax polymers containing glutamate receptor antagonists. J Neurosci Methods 60:211-217.

Stevenson JA, Lund RD (1982) Alterations of the crossed parabigeminotectal projection induced by neonatal eye removal in rats. J Comp Neurol 207:191-202.

Thompson I, Holt C (1989) Effects of intraocular tetrodotoxin on the development of the retinocollicular pathway in the Syrian hamster. J Comp Neurol 282:371-388.

Thong IG, Dreher B (1986) The development of the corticotectal pathway in the albino rat. Brain Res 390:227-238.

Udin SB, Scherer WJ (1990) Restoration of the plasticity of binocular maps by NMDA after the critical period in Xenopus. Science 249:669-672.

Vercelli A, Garbossa D, Biasiol S, Repici M, Jhaveri S (2000) NOS inhibition during postnatal development leads to increased ipsilateral retinocollicular and retinogeniculate projections in rats. Eur J Neurosci 12:473-490. 